\title{
RESPUESTA DEL Eucalyptus globulus ssp. globulus A LA PREPARACION DEL SITIO, CONTROL DE MALEZAS Y ENMIENDAS NUTRICIONALES
}

Sergio Calderón (")

\section{RESUMEN}

El informe evalúa la respuesta volumétrica y financiera del Eucalyptus globulus ssp. globulus a la preparación del sitio, analizando el efecto del método de plantación, el control de malezas y tratamientos de fertilización, en la zona costera-sur de la provincia de Concepción.

El estudio analiza los resultados con certeza estadística, establece las ganancias volumétricas, las proyecta a la edad de rotación y efectúa un análisis económico marginal de ellas.

La mejor alternativa desde el punto de vista del método de plantación resulta ser la ahoyadura con pala (tradicional) que supera significativamente al de motoperforadora $y$, aun cuando sin significación, al tratamiento de surcos y casillas.

Las plantaciones espaciadas a $3 \times 3 \mathrm{~m}$, usando herbicida (Round-up) y fertilizante (urea, superfosfato y borax), son mejores que las plantaciones fertilizadas y espaciadas a $2 \times 2 \mathrm{~m}$, tanto por generar un mayor volumen como por representar un menor costo marginal. Estas dos alternativas tienen una rentabilidad superior al $12 \%$, si se considera un precio real de mercado equivalente a US\$ $12 / \mathrm{m}^{3}$ para la madera en pie.

Palabras Claves: Eucalyptus globulus ssp globulus. Preparación del Sitio. Herbicidas. Fertilizantes. 


\section{ABSTRACT}

This report shows the volumetric and financial response of Eucalyptus globulus ssp. globulus growing in plantation established in the southern-costal zone in Concepcion Province (Chile), when the site productivity is modified by testing different combinations of planting methods, weөd control and/or fertilizing treatments and established with different initial stockings.

The study analizes the results with statistical certainty, it specifies the actual volumetric gains that are projected to rotation ages and allow marginal economic analysis from them.

The best establishment method is the traditional technique preparing holes using hand tools that is significantly different to motordrilled holes and better, but no significant, than plow trench and cultivated box.

The composite treatment of spacing by $3 \times 3 \mathrm{~m}$, herbicide (Round-up) and fertilizer mix (urea, superphospate, borax) is better than $2 \times 2 \mathrm{~m}$ spacing (with fertilizer only) in volume and financially. These two alternatives have a profitability greater than $12 \%$ when the stumpage market price is higher than US $\$ 12 / \mathrm{m}^{3}$.

Keywords: Eucalyptus globulus ssp. globulus. Site preparation. Herbicides. Fertilizer. 


\section{INTRODUCCION}

Las plantas recién establecidas compiten con las malezas para aprovechar la luz, nutrientes y agua y es el nivel de fertilidad del suelo el factor principal que regula la intensidad de esta lucha y determina el sentido en que se inclinará el balance. Evidentemente todo mejoramiento de las técnicas de preparación del sitio que eleve el nivel competitivo de las plantas, co-ayuda al establecimiento de la plantación. Numerosos ensayos establecidos en diversas localidades permiten evaluar la respuesta al uso de herbicidas, como herramienta para suprimir o atenuar esta competencia, y permiten establecer los efectos e interacciones que se introducen, al cambiar la productividad del sitio con enmiendas de fertilizantes y preparación del sitio.

Existen resultados de estos ensayos para las zonas áridas en Illapel, IV Región (Wrann e Infante, 1988), semiáridas en Casablanca, V Región (Prado y rojas, 1987) y Santa Cruz, VI Región. En la zona costera-sur de la Provincia de Concepción, en tanto, existen resultados hasta el año 1988, los cuales se actualizan en este artículo.

En la mayoria de los lugares mencionados existen ensayos que incluyen preparación de sitio, herbicida y fertilizante, generalmente con un diseño factorial. En el caso de la Provincia de Concepción se dispone de dos ensayos separados, en el primero de los cuales se prueban métodos de preparación de suelos, adecuados para topografias quebradas donde la mecanización es dificil o imposible, y, en el segundo, diferentes combinaciones de herbicidas y fertilizantes, además de diferentes espaciamientos de plantación.

El estudio de resultados del ensayo de herbicida y fertilizantes en plantaciones de distinta densidad, considera sólo dos de los espaciamientos. En cada caso, se buscan las diferencias significativas entre tratamientos, mediante un Análisis de Varianza y las consiguientes pruebas de Tukey. Los resultados en crecimiento diametral y en altura, son proyectados hasta edades de rotación de 10 y 15 años utilizando un modelo de simulación del crecimiento representativo de las plantaciones del norte de Tasmania (Goodwin N. y Candy S.G., 1986) Comparando los resultados de tratamientos especificos con el testigo se obtienen las ganancias volumétricas que, una vez establecido su 
costo, pueden ser valoradas para diferentes tasas de rentabilidad al nivel mínimo de equilibrio.

\section{MATERIAL Y METODO}

En Colcura, Comuna de Lota (Provincia de Concepción) se establecieron dos ensayos, uno para probar técnicas de preparación de suelo y otro para determinar el efecto de la aplicación de herbicida y fertilizante.

El primer experimento, establecido en 1986, tiene un diseño de bloques al azar con tres repeticiones, considerando los siguientes tratamientos :

1) Ahoyadura con pala (testigo)

2) Casilla de $50 \times 50 \mathrm{~cm}$.

3) Ahoyadura con motoperforadora

4) Surcos

La técnica de ahoyadura con pala que se usa tradicionalmente en el país, fue elegida como patrón de comparación. El segundo tratamiento consiste en una preparación de suelo en un área de $50 \times 50 \mathrm{~cm}$ con $30 \mathrm{~cm}$ de profundidad. El hoyo efectuado con motoperforadora alcanzó una profundidad promedio de $50 \mathrm{~cm}$. El segundo experimento se estableció de acuerdo a un diseño completamente aleatorizado y tiene como objetivo analizar, para 3 espaciamientos distintos, el efecto de la aplicación de herbicida, fertilizante y la combinación de ambos. El ensayo fue establecido en 1984, en un sector bajo, con una napa freática cercana a la superficie (vega) donde predominaba una vegetación herbácea perenne. La plantación se realizó empleando una pala de media caña.

Los espaciamientos representados son $2 \times 2 \mathrm{~m} ; 3 \times 3 \mathrm{~m}$ y $4 \times 4 \mathrm{~m}$, combinados con los siguientes tratamientos :

1) Testigo (sin aplicación)

2) Aplicación de herbicida

3) Aplicación de fertilizante

4) Aplicación de herbicida y fertilizante

Las parcelas son de $\mathbf{4 9}$ plantas, de las cuales se controlan las 25 interiores. 
El herbicida (Glyphosate, 4 L/ha) se aplicó después de la plantación en una faja de $1 \mathrm{~m}$ de ancho, cubriendo las plantas al momento de la aplicación.

El fertilizante, que consistió de $75 \mathrm{~g}$ de superfosfato triple, $158 \mathrm{~g}$ de Urea y $54 \mathrm{~g}$ de borax por planta, se aplic6 en 3 oportunidades : $20 \%$ al momento de plantar, $40 \%$ después de 1 año y $40 \%$ después de 2 años.

El estudio estadístico incluye un análisis de varianza (ANDEVA) que establece si las diferencias entre tratamiento son significativas y distribuye la varianza a sus diferentes causas : tratamientos, bloques y error experimental. La prueba adicional de Tukey especifica la significación que existe entre cada tratamiento y permite seleccionar el mejor, según la variable analizada.

En el segundo ensayo, los datos observados al quinto año son proyectados a los 10 y 15 años con un modelo de crecimiento para plantaciones de Eucalyptus globulus desarrollado en Tasmania (Goodwin N. y Candy S.G., 1986), el cual no ha sido validado para las condiciones chilenas. La proyección se incluye con carácter demostrativo solamente, dado que la representatividad debe primero probarse. Sin embargo, en el presente estudio se considera que cierta similitud en el volumen a los $\mathbf{5}$ años entre el modelo australiano y el promedio de 2 funciones chilenas para la zona, es garantia de igualdad en el crecimiento hasta esa edad (Apéndice 1).

El análisis económico se aborda con caracter marginal, capitalizando hasta la edad de rotación solo los costos imputables a los tratamientos aplicados. Estos se comparan con los ingresos también marginales generados por la venta del excedente de volumen producido por los tratamientos en relación al testigo.

\section{RESULTADOS Y DISCUSION}

\section{Métodos de Plantación}

El efecto de la preparación del suelo en el crecimiento del E. globulus puede ser observado en todas las variables medidas, aun cuando no sea significativo en todos los casos. El Cuadro $\mathrm{N}^{\circ} 1$ muestra el estado de estas variables a los 3 años de edad. 
El efecto de los tratamientos se refleja tanto en el crecimiento diamétrico, la altura total del árbol y la supervivencia de las plantas. Los controles efectuados a distintas edades se consideran valiosos para reflejar el efecto de los tratamientos después de transcurrido un lapso de 2 años desde su instalación o aplicación. Los controles efectuados al tercer año cumplen con este requisito. También se incluye el calculo de un índice $\left(D^{2} \times H\right)$ combinando las variables D.A.P. y Altura Total, el cual tiene el potencial de expresar en mejor forma el crecimiento volumétrico.

La única tendencia que se puede observar a priori es que el tratamiento de ahoyadura con motoperforadora presenta el efecto de reducir el diámetro, la altura y el indice $D^{2} \times \mathrm{H}$ comparando los resultados con los otros tratamientos. El análisis de varianza, con respecto a la variable altura, confiere seguridad estadística a esta afirmación y la prueba de comparación múltiple de Tukey (Cuadro $\mathrm{N}^{\circ} 2$ ) individualiza los tratamientos con diferencias significativas.

\section{Cuadro $N^{0} 1$}

RESUltados del METOdo de PLANTACION A LOS 3 AÑos

\begin{tabular}{|c|c|c|c|c|c|}
\hline Tratamiento & Bloque & $\begin{array}{l}\text { Diametro } \\
(\mathrm{cm})\end{array}$ & $\begin{array}{c}\text { Altura } \\
\text { (m) }\end{array}$ & $\begin{array}{l}D^{2} \times \mathrm{H} \\
\left(\mathrm{cm}^{3}\right) \\
\end{array}$ & $\begin{array}{c}\text { Supervivencia. } \\
\text { (\%) }\end{array}$ \\
\hline $\begin{array}{ll} & 1 \\
& 1 \\
& 1 \\
\text { Pala } \quad\end{array}$ & $\begin{array}{l}1 \\
2 \\
3\end{array}$ & $\begin{array}{l}6,34 \\
7,23 \\
6,40 \\
6,65\end{array}$ & $\begin{array}{l}7,35 \\
7,34 \\
7,24 \\
7,31\end{array}$ & $\begin{array}{l}31.703,0 \\
48.671,8 \\
36.566,9 \\
38.980,6\end{array}$ & $\begin{array}{l}84 \\
92 \\
92 \\
89\end{array}$ \\
\hline $\begin{array}{lr} & 2 \\
& 2 \\
& 2 \\
\text { Casilla } & \\
\end{array}$ & $\begin{array}{l}1 \\
2 \\
3\end{array}$ & $\begin{array}{l}6,16 \\
6,70 \\
7,61 \\
6,82 \\
\end{array}$ & $\begin{array}{l}7,13 \\
6,75 \\
7,85 \\
7,24 \\
\end{array}$ & $\begin{array}{l}35.726,9 \\
37.833,4 \\
55.365,4 \\
42.975,2 \\
\end{array}$ & $\begin{array}{l}88 \\
84 \\
80 \\
84\end{array}$ \\
\hline $\begin{array}{c}3 \\
3 \\
3 \\
\text { M. Perforadora }\end{array}$ & $\begin{array}{l}1 \\
2 \\
3\end{array}$ & $\begin{array}{l}4,40 \\
5,84 \\
5,45 \\
5,23 \\
\end{array}$ & $\begin{array}{l}4,73 \\
6,00 \\
5,80 \\
5,51\end{array}$ & $\begin{array}{l}15.439,5 \\
24.853,8 \\
20.147,7 \\
20.147,0\end{array}$ & $\begin{array}{l}80 \\
96 \\
64 \\
80\end{array}$ \\
\hline $\begin{array}{ll} & 4 \\
& 4 \\
& 4 \\
\text { Surco } & \\
\end{array}$ & $\begin{array}{l}1 \\
2 \\
3\end{array}$ & $\begin{array}{l}6,31 \\
6,03 \\
7,76 \\
6,70 \\
\end{array}$ & $\begin{array}{l}6,85 \\
6,23 \\
8,17 \\
7,08 \\
\end{array}$ & $\begin{array}{l}34.247,7 \\
31.611,3 \\
54.616,5 \\
40.158,5\end{array}$ & $\begin{array}{l}88 \\
76 \\
76 \\
80\end{array}$ \\
\hline
\end{tabular}




\section{Cuadro $\mathrm{N}^{\circ} 2$}

PRUEBA DE TUKEY

COMPARACION MULTIPLE DE LA ALTURA

\begin{tabular}{|c|c|c|c|c|}
\hline $\begin{array}{c}\text { Tratamiento } \\
\text { Promedio }\end{array}$ & 1 & 2 & 3 & 4 \\
\hline 1 & 0,3 & 7,2 & 7,1 & 5,5 \\
\hline 2 & 0,0 & 0,1 & 0,2 & $1,8^{*}$ \\
4 & 0,0 & 0,0 & $1,7^{*}$ \\
3 & 0,0 & 0,0 & 0,0 & 1,6 \\
\hline
\end{tabular}

Nota: Valores con asterisco $\left({ }^{*}\right)$, son significativamente diferentes entre si al nivel del $95 \%$.

La prueba de Tukey confirma que la ahoyadura con motorperforadora produce un significativo deterioro de la altura en relación a la ahoyadura con pala (tradicional) y el método con casillas. La razón del bajo comportamiento del tratamiento con motorperforadora es que la máquina al abrir el hoyo de plantación, produce un efecto de compactación y sellado de las paredes laterales por la fuerte presión aplicada, la cual se hace más notoria cuando aumenta la proporción de arcillas y existe cierta humedad en el suelo.

\section{Espaciamiento}

El efecto del espaciamiento en los tratamientos de herbicida y fertilizante es ya mensurable a los 5 años de edad. No cabe duda que este efecto se hará más notorio con la maduración de la plantación.

Los promedios por espaciamientos para todos los tratamientos aparecen en el Cuadro $\mathrm{N}^{\circ} 3$. 


\section{Cuadro $\mathrm{N}^{\circ} 3$}

PARAMETROS MEDIOS POR ESPACIAMIENTO 6 AÑOS DE EDAD

\begin{tabular}{|c|c|c|}
\hline $\begin{array}{c}\text { Espaciamiento } \\
(\mathrm{m})\end{array}$ & $\begin{array}{c}\text { Diametro } \\
(\mathrm{cm})\end{array}$ & $\begin{array}{c}\text { Altura } \\
(\mathrm{m})\end{array}$ \\
\hline $2 \times 2$ & 6,48 & 9,12 \\
$3 \times 3$ & 9,81 & 11,46 \\
$4 \times 4$ & 9,86 & 10,62 \\
\hline
\end{tabular}

Desde ya se puede observar que los árboles de mayor espaciamiento ( $3 \times 3$, $4 \times 4 \mathrm{~m}$ ) están creciendo mejor en diámetro. Esto indica que a las más altas densidades $(2 \times 2 \mathrm{~m})$ la competencia ya ha comenzado. Sin embargo, el crecimiento en altura aunque tiende a aumentar al bajar la competencia desde espaciamientos de $2 \times 2$ a $3 \times 3 \mathrm{~m}$, revierte esta tendencia cuando los espaciamientos se hacen suficientemente grandes para permitir el crecimiento libre y sin competencia.

Estos crecimientos diferenciados por el cambio del factor espaciamiento, interactuan y modifican desde el punto volumétrico y económico, los resultados obtenidos por los otros tratamientos aplicados : herbicida y fertilizante.

\section{Herbicida y Fertilizante}

Las consecuencias del uso de herbicida y/o fertilizante en plantaciones de E. globulus establecidas con ahoyadura con pala y con distinto espaciamiento, se muestran a los 5 años de edad. En el Cuadro $\mathrm{N}^{\circ} 4$ se presentan los resultados de los espaciamientos $2 \times 2$ y $3 \times 3 \mathrm{~m}$. Por ser estos los más usados en la práctica son también los únicos sometidos a análisis económico. 


\section{Cuadro $\mathrm{N}^{\circ} 4$}

\section{RESULTADOS DEL ENSAYO DE USO DE HERBICIDAS Y FERTILIZANTES EN PLANTACIONES CON ESPACIAMIENTO $2 \times 2 \mathrm{~m} Y 3 \times 3 \mathrm{~m}$}

\begin{tabular}{|c|c|c|c|c|c|c|}
\hline Tratamiento & $\begin{array}{c}\text { Espac. } \\
\text { (m) }\end{array}$ & Bloque & $\begin{array}{c}\text { Diametro } \\
(\mathrm{cm})\end{array}$ & $\begin{array}{l}\text { Altura } \\
\text { (m) }\end{array}$ & $\begin{array}{l}D^{2} \times H \\
\left(\mathrm{~cm}^{3}\right)\end{array}$ & $\begin{array}{c}\text { Superv. } \\
(\%)\end{array}$ \\
\hline $\begin{array}{r}1 \\
1 \\
1 \\
\text { Testigo }\end{array}$ & $\begin{array}{l}2 \times 2 \\
2 \times 2 \\
2 \times 2\end{array}$ & $\begin{array}{l}1 \\
2 \\
3\end{array}$ & $\begin{array}{l}7,3 \\
7,6 \\
8,5 \\
7,8\end{array}$ & $\begin{array}{r}9,7 \\
10,1 \\
10,7 \\
10,2\end{array}$ & $\begin{array}{l}62.644 \\
72.547 \\
94.732 \\
76.641\end{array}$ & $\begin{array}{l}80 \\
80 \\
76 \\
79\end{array}$ \\
\hline $\begin{array}{r}2 \\
2 \\
2 \\
\text { Herbicida } \\
\end{array}$ & $\begin{array}{l}2 \times 2 \\
2 \times 2 \\
2 \times 2\end{array}$ & $\begin{array}{l}1 \\
2 \\
3\end{array}$ & $\begin{array}{l}7,6 \\
7,6 \\
7,6 \\
7,6 \\
\end{array}$ & $\begin{array}{l}11,5 \\
11,5 \\
11,5 \\
11,5 \\
\end{array}$ & $\begin{array}{l}85.841 \\
85.000 \\
85.000 \\
85.280 \\
\end{array}$ & $\begin{array}{l}88 \\
80 \\
80 \\
83 \\
\end{array}$ \\
\hline $\begin{array}{c}3 \\
3 \\
3 \\
\text { Fertilizante }\end{array}$ & $\begin{array}{l}2 \times 2 \\
2 \times 2 \\
2 \times 2\end{array}$ & $\begin{array}{l}1 \\
2 \\
3\end{array}$ & $\begin{array}{r}8,9 \\
10,8 \\
9,0 \\
9,6\end{array}$ & $\begin{array}{l}13,2 \\
14,1 \\
13,5 \\
13,6\end{array}$ & $\begin{array}{l}128.800 \\
188.000 \\
150.000 \\
155.600\end{array}$ & $\begin{array}{l}80 \\
80 \\
80 \\
80\end{array}$ \\
\hline $\begin{array}{r}4 \\
4 \\
4 \\
\text { Herbi-Fert }\end{array}$ & $\begin{array}{l}2 \times 2 \\
2 \times 2 \\
2 \times 2\end{array}$ & $\begin{array}{l}1 \\
2 \\
3\end{array}$ & $\begin{array}{l}9,1 \\
8,8 \\
8,5 \\
8,8\end{array}$ & $\begin{array}{l}13,6 \\
12,9 \\
13,1 \\
13,2\end{array}$ & $\begin{array}{l}149.600 \\
129.100 \\
113.800 \\
130.800\end{array}$ & $\begin{array}{r}100 \\
88 \\
96 \\
95\end{array}$ \\
\hline $\begin{array}{r}1 \\
1 \\
1 \\
\text { Testigo }^{1}\end{array}$ & $\begin{array}{l}3 \times 3 \\
3 \times 3 \\
3 \times 3\end{array}$ & $\begin{array}{l}1 \\
2 \\
3\end{array}$ & $\begin{array}{l}8,3 \\
7,8 \\
9,1 \\
8,4\end{array}$ & $\begin{array}{r}10,9 \\
9,4 \\
10,8 \\
10,4\end{array}$ & $\begin{array}{l}83.013 \\
63.956 \\
98.765 \\
81.911 \\
\end{array}$ & $\begin{array}{l}72 \\
80 \\
96 \\
83 \\
\end{array}$ \\
\hline $\begin{array}{r}2 \\
2 \\
2 \\
\text { Herbicida }\end{array}$ & $\begin{array}{l}3 \times 3 \\
3 \times 3 \\
3 \times 3\end{array}$ & $\begin{array}{l}1 \\
2 \\
3\end{array}$ & $\begin{array}{r}11,3 \\
8,5 \\
7,7 \\
9,1\end{array}$ & $\begin{array}{r}12,7 \\
10,9 \\
9,1 \\
10,9\end{array}$ & $\begin{array}{r}197.365 \\
96.715 \\
69.635 \\
121.238\end{array}$ & $\begin{array}{r}100 \\
80 \\
96 \\
92\end{array}$ \\
\hline $\begin{array}{c}3 \\
3 \\
3 \\
\text { Fertilizante }\end{array}$ & $\begin{array}{l}3 \times 3 \\
3 \times 3 \\
3 \times 3\end{array}$ & $\begin{array}{l}1 \\
2 \\
3\end{array}$ & $\begin{array}{r}9,3 \\
9,9 \\
10,8 \\
10,0\end{array}$ & $\begin{array}{l}11,2 \\
11,2 \\
12,8 \\
11,7\end{array}$ & $\begin{array}{l}105.781 \\
119.589 \\
165.566 \\
130.312\end{array}$ & $\begin{array}{l}88 \\
92 \\
96 \\
92\end{array}$ \\
\hline $\begin{array}{c}4 \\
4 \\
4 \\
\text { Herbi-Fert }\end{array}$ & $\begin{array}{l}3 \times 3 \\
3 \times 3 \\
3 \times 3\end{array}$ & $\begin{array}{l}1 \\
2 \\
3\end{array}$ & $\begin{array}{l}12,1 \\
10,2 \\
12,2 \\
11,5\end{array}$ & $\begin{array}{l}13,9 \\
10,5 \\
13,7 \\
12,7\end{array}$ & $\begin{array}{l}236.472 \\
140.628 \\
221.687 \\
199.596\end{array}$ & $\begin{array}{l}96 \\
96 \\
88 \\
93\end{array}$ \\
\hline
\end{tabular}

La aplicación de los tratamientos herbicida y fertilización produce un mejoramiento del crecimiento diametral, en altura, del indice $D^{2} \times \mathrm{H}$ y de la supervivencia, ya sea cuando los tratamientos se aplican por separado, 0 cuando se consideran conjuntamente. 
Generalmente la aplicación de ambos multiplica el beneficio. La única excepción a esta regla la constituye el uso exclusivo de fertilizante, que en las plantaciones de $2 \times 2 \mathrm{~m}$ supera levemente al tratamiento combinado con herbicida, lo cual se considera anormal, puesto que el herbicida ayuda a suprimir la competencia de las malezas.

Todas las conclusiones poseen una alta validez estadistica, pues de 24 veces que se combinan las 4 variables medidas con los tratamientos herbicidafertilizante y fertilizante en los 3 espaciamientos ensayados, los resultados son 24 veces favorables y 13 veces significativos.

\section{Estimación de la Ganancia Volumétrica Futura}

Para apreciar la ganancia producida por la aplicación de los tratamientos es interesante expresarla en volumen, que combina el efecto del diámetro y altura. Cuando este volumen se expresa por hectárea también incluye el mejoramiento de la supervivencia. Comparando el volumen por tratamiento con el testigo (sin tratamiento) se puede obtener la ganancia marginal producida por el tratamiento. Lo importante es proyectar esta diferencia hasta la edad de cosecha final, edad a la cual se producen los ingresos y hasta la cual deben ser capitalizados los costos.

La proyección del crecimiento volumétrico en rotaciones de 10 y 15 años se presenta para las plantaciones de $2 \times 2 \mathrm{~m}$ con aplicación exclusiva de fertilizante en el Cuadro $\mathrm{N}^{\circ} 5$ y para las plantaciones de $3 \times 3 \mathrm{~m}$ con aplicación combinada de herbicida y fertilizante en el Cuadro $\mathrm{N}^{\circ} 6$. La misma información se presenta en forma esquemática en la Figura $\mathrm{N}^{\circ} 1$. 


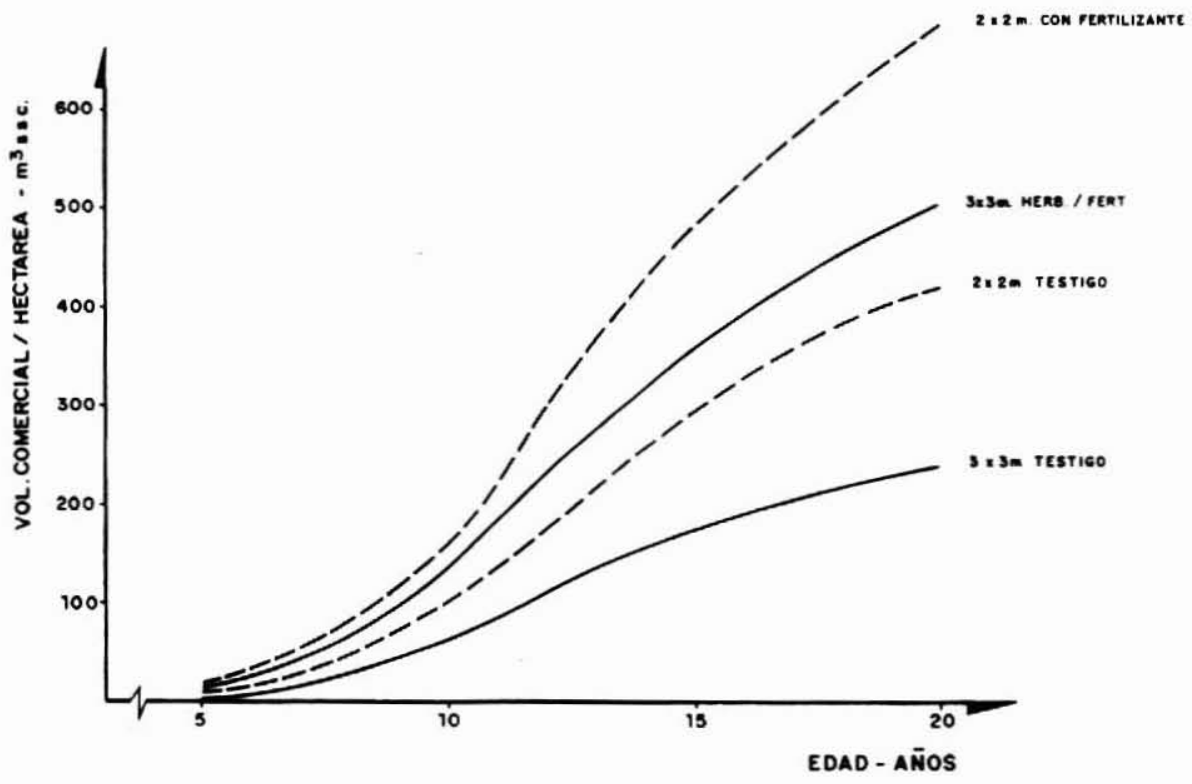

Figura N ${ }^{\circ}$ 1. PROYECCION DEL CRECIMIENTO EN PLANTACIONES DE $2 \times 2 \mathrm{~m}$ CON Y SIN USO DE FERTILIZANTE 


\section{Cuadro $\mathrm{N}^{\circ} 5$ \\ PROYECCION DEL CRECIMIENTO EN PLANTACIONES DE $2 \times 2 \mathrm{~m}$ CON Y SIN USO DE FERTILIZANTE}

\begin{tabular}{|c|c|c|c|c|}
\hline Variable & Edad 5 & Edad 10 & Edad 12 & Edad 15 \\
\hline $\begin{array}{l}\text { Fertilizante } \\
\text { D.A.P. }(\mathrm{cm}) \\
\text { Altura }(\mathrm{m}) \\
\text { Vol/arb }\left(\mathrm{m}^{3} \mathrm{ssc}\right) \\
\text { Densidad/ha } \\
\text { Vol totha }\left(\mathrm{m}^{3} \mathrm{ssc}\right) \\
\text { Vol com/ha }\left(\mathrm{m}^{3} \mathrm{ssc}\right)\end{array}$ & $\begin{array}{r}9,6 \\
13,6 \\
0,0225 \\
2067 \\
46,5 \\
19,5 \\
\end{array}$ & $\begin{array}{r}14,2 \\
27,7 \\
0,1002 \\
2067 \\
207,1 \\
159,5 \\
\end{array}$ & $\begin{array}{r}15,2 \\
31,5 \\
0,1910 \\
2004 \\
382,8 \\
306,2 \\
\end{array}$ & $\begin{array}{r}16,8 \\
36,0 \\
0,2850 \\
1915 \\
545,8 \\
480,3\end{array}$ \\
\hline $\begin{array}{l}\text { Testigo } \\
\text { D.A.P. (cm) } \\
\text { Altura }(\mathrm{m}) \\
\text { Vol/arb }\left(\mathrm{m}^{3} \mathrm{ssc}\right) \\
\text { Densidad } / \mathrm{ha} \\
\text { Vol tot/ha }\left(\mathrm{m}^{3} \mathrm{ssc}\right) \\
\text { Vol com/ha }\left(\mathrm{m}^{3} \mathrm{ssc}\right)\end{array}$ & $\begin{array}{r}7,8 \\
10,2 \\
0,0111 \\
1968 \\
21,8 \\
6,1 \\
\end{array}$ & $\begin{array}{r}11,5 \\
27,7 \\
0,0904 \\
1968 \\
177,9 \\
101,4 \\
\end{array}$ & $\begin{array}{r}12,4 \\
31,5 \\
0,1270 \\
1968 \\
249,9 \\
167,4 \\
\end{array}$ & $\begin{array}{r}13,7 \\
36,0 \\
0,1878 \\
1828 \\
343,2 \\
295,2\end{array}$ \\
\hline $\begin{array}{l}\text { Diferencia } \\
\text { Vol/ha }\left(m^{3} s s c\right)\end{array}$ & 13,4 & 58,1 & 138,8 & 185,0 \\
\hline
\end{tabular}

En las plantaciones espaciadas $2 \times 2 \mathrm{~m}$, el volumen comercial obtenido con la aplicación de fertilizantes es $\mathbf{5 7 . 3 \%}$ mayor al testigo cuando este se proyecta al año 10.

El incremento del volumen comercial es similar $(62.7 \%)$ cuando la proyección se hace hasta una edad de rotación de 15 años.

El tratamiento de mejor rendimiento en las plantaciones de 5 años de edad con espaciamiento de $3 \times 3 \mathrm{~m}$, resulta ser el que combina las aplicaciones de herbicida y fertilizantes en la etapa de establecimiento. En el Cuadro $\mathrm{N}^{\circ} 6 \mathrm{se}$ presentan los resultados también proyectados a las edades de rotación 10 y 15 años.

Cuando el E. globulus es plantado con un espaciamiento de $3 \times 3 \mathrm{~m}$ y se trata el sitio inicialmente con herbicida y fertilizante en los 3 primeros años de establecimiento, la ganancia volumétrica por hectárea, proyectada al año 10, es de un $107.3 \%$ lo cual indica un mejoramiento substancial.

Este aumento es de un $115.2 \%$ cuando la proyección se hace al año 15. 


\section{Cuadro $\mathrm{N}^{\circ} 6$}

PROYECCION DEL CRECIMIENTO EN PLANTACIONES DE $3 \times 3 \mathrm{~m}$ CON Y SIN USO DE HERBICIDA-FERTILIZANTE

\begin{tabular}{|c|c|c|c|c|}
\hline Variable & Edad 5 & Edad 10 & Edad 12 & Edad 15 \\
\hline $\begin{array}{l}\text { Herb - Fert } \\
\text { D.A.P. (cm) } \\
\text { Altura }(\mathrm{m}) \\
\text { Vol/arb }\left(\mathrm{m}^{3} \mathrm{ssc}\right) \\
\text { Densidad/ha } \\
\text { Vol totha }\left(\mathrm{m}^{3} \mathrm{ssc}\right) \\
\text { Vol com/ha }\left(\mathrm{m}^{3} \mathrm{ssc}\right)\end{array}$ & $\begin{array}{r}11,6 \\
12,7 \\
0,0305 \\
1037 \\
31,6 \\
18,0 \\
\end{array}$ & $\begin{array}{r}17,1 \\
27,7 \\
0,1998 \\
1037 \\
207,2 \\
136,8 \\
\end{array}$ & $\begin{array}{r}18,5 \\
31,2 \\
0,2806 \\
1000 \\
280,6 \\
246,9 \\
\end{array}$ & $\begin{array}{r}20,2 \\
36,0 \\
0,4126 \\
966 \\
398,6 \\
358,7 \\
\end{array}$ \\
\hline $\begin{array}{l}\text { Testigo } \\
\text { D.A.P. (cm) } \\
\text { Altura }(\mathrm{m}) \\
\text { Vol/arb }\left(\mathrm{m}^{3} \mathrm{ssc}\right) \\
\text { Densidad/ha } \\
\text { Vol tot/ha }\left(\mathrm{m}^{3} \mathrm{ssc}\right) \\
\text { Vol com/ha }\left(\mathrm{m}^{3} \mathrm{ssc}\right)\end{array}$ & $\begin{array}{r}8,5 \\
10,4 \\
0,0133 \\
919 \\
12,2 \\
4,3 \\
\end{array}$ & $\begin{array}{r}12,5 \\
27,4 \\
0,1056 \\
919 \\
97,1 \\
66,0 \\
\end{array}$ & $\begin{array}{r}13,6 \\
29,6 \\
0,1408 \\
919 \\
129,4 \\
97,0 \\
\end{array}$ & $\begin{array}{r}14,8 \\
36,0 \\
0,2195 \\
841 \\
184,6 \\
166,7 \\
\end{array}$ \\
\hline $\begin{array}{l}\text { Diferencia } \\
\text { Vol/ha }\left(\mathrm{m}^{3} \mathrm{ssc}\right) \\
\end{array}$ & 13,7 & 70,8 & 149,9 & 192,0 \\
\hline
\end{tabular}

\section{Análisis Económico Marginal}

Plantaciones Espaciadas $2 \times 2 m$ y Fertilizadas

El análisis económico marginal del mejor tratamiento (Cuadro $\mathrm{N}^{\circ} 7$ ) deberia representar también la mejor solución económica. Sin embargo, no siempre lo es. Hay ocasiones en que la reducción de costos de un tratamiento es suficientemente importante como para contrarrestar un incremento volumétrico menor. En este caso particular la aplicación de fertilizante es el tratamiento de menor costo, además de ser el de mayor incremento volumétrico. Por lo tanto es la solución económica marginal más eficiente.

Los resultados (tasas de cambio a Julio de 1991) muestran que el mercado debe alcanzar precios de madera en pie de $\$ 2.148 / \mathrm{m}^{3}$ (US $\$ 6.14 / \mathrm{m}^{3}$ ) para que el tratamiento con fertilizantes sea económico y rinda un $8 \%$ de beneficio cuando las plantaciones establecidas a $2 \times 2 \mathrm{~m}$ se manejan sin raleo y con corta final a los 15 años. Si la corta final se realiza a los 10 años, el precio debe 
subir a $\$ 4.656 / \mathrm{m}^{3}$ (US $\$ 13.30 / \mathrm{m}^{3}$ ) para rendir el mismo interés.

\section{Cuadro $\mathrm{N}^{\circ} 7$}

\section{ANALISIS FINANCIERO MARGINAL PARA EL TRATAMIENTO DE FERTILIZACION EN SUELO DE VEGA $(2 \times 2 \mathrm{~m})$}

\begin{tabular}{|c|c|c|c|c|c|c|}
\hline \multirow[t]{2}{*}{ Año } & \multirow[t]{2}{*}{ Gastos de Operación } & \multirow{2}{*}{$\begin{array}{l}\text { Costo } \\
\text { Actual } \\
\text { (\$/ha) }\end{array}$} & \multicolumn{2}{|c|}{$\begin{array}{c}\text { Costo } \\
\text { Ano } 10 \\
\end{array}$} & \multicolumn{2}{|c|}{$\begin{array}{c}\text { Capitalizado } \\
\text { Ano } 15\end{array}$} \\
\hline & & & $8 \%$ & $12 \%$ & $8 \%$ & $12 \%$ \\
\hline 0 & $\begin{array}{l}20 \% \text { dosis fertilizante } \\
2.25 \text { jornadas+leyes soc. } \\
79 \mathrm{Kg} / \mathrm{ha} \text { Urea }(\$ 96 / \mathrm{Kg}) \\
37.5 \mathrm{Kg} / \mathrm{ha} \text { Super } \mathrm{f} \text { triple } \\
27 \mathrm{Kg} \text {.ha Borax }(181 / \mathrm{Kg}) \\
1 / 20 \text { jornadas de superv. }\end{array}$ & $\begin{array}{l}9.000 \\
7.584 \\
3.188 \\
4.887 \\
1.012\end{array}$ & & & & \\
\hline 1 & $\begin{array}{l}40 \% \text { dosis fertilizante } \\
4.5 \text { jornadas+leyes soc. } \\
158 \mathrm{~g} / \mathrm{ha} \text { Urea }(\$ 96 / \mathrm{Kg}) \\
75 \mathrm{Kg} / \text { ha Super } \mathrm{f} \text { triple } \\
54 \mathrm{Kg} / \mathrm{ha} \text { Borax }(181 / \mathrm{Kg}) \\
1 / 20 \text { jornadas de superv. }\end{array}$ & $\begin{array}{r}25.671 \\
18.000 \\
15.168 \\
6.376 \\
9.774 \\
1.350\end{array}$ & 55.422 & 79.730 & 81.433 & 140.512 \\
\hline \multirow[t]{2}{*}{2} & $\begin{array}{l}40 \% \text { dosis fertilizante } \\
4.5 \text { jornadas }+ \text { leyes soc. } \\
158 \mathrm{~g} / \mathrm{ha} \text { Urea }(\$ 96 / \mathrm{Kg}) \\
75 \mathrm{Kg} / \text { ha Super } \mathrm{f} \text { triple } \\
54 \mathrm{Kg} / \mathrm{ha} \text { Borax }(181 / \mathrm{Kg}) \\
1 / 20 \text { jornadas de superv. }\end{array}$ & $\begin{array}{r}50.668 \\
18.000 \\
15.168 \\
6.376 \\
9.774 \\
1.350\end{array}$ & 101.286 & 140.506 & 148.822 & 247.620 \\
\hline & & 50.668 & 93.783 & 125.452 & 137.798 & 221.089 \\
\hline & & Gran Total & 250.491 & 345.688 & 368.053 & 609.221 \\
\hline \multicolumn{2}{|c|}{$\begin{array}{l}\text { Costo de equilibrio } \$ / \mathrm{m}^{3} \text { en pie } \\
\text { Beneficio Neto=tasa de interés }\end{array}$} & & $\begin{array}{r}4.311 \\
+345\end{array}$ & $\begin{array}{r}5.950 \\
714\end{array}$ & $\begin{array}{r}1.989 \\
159\end{array}$ & $\begin{array}{r}3.293 \\
395\end{array}$ \\
\hline \multicolumn{2}{|c|}{ Precio de Equilibrio \$//3 en pie } & & 4.656 & 6.664 & 2.148 & 3.688 \\
\hline
\end{tabular}

Asimismo, si el precio de mercado para la madera en pie es de un valor de $\$ 3.688 / \mathrm{m}^{3}$ (US\$ $10.54 / \mathrm{m}^{3}$ ) y se ha esperado 15 años para alcanzar la madurez, la renta lograda en la gestión económica con aplicación de fertilizantes es de un $12 \%$. Si el tiempo de espera, con las mismas condiciones de manejo se reduce a 10 años, el valor de la madera en pie sube a $\$ 6.664 / \mathrm{m}^{3}$ (US\$ $19.04 / \mathrm{m}^{3}$ ) para rendir una Tasa Interna de Retorno (TIR) de $12 \%$. 
Plantaciones Espaciadas $3 \times 3 \mathrm{~m}$ y tratadas con Herbicida

En las plantaciones espaciadas $3 \times 3 \mathrm{~m}$ el tratamiento de mayor rendimiento resulta ser el que combina el uso de herbicida y fertilizante, situación que no difiere de los resultados alcanzados en el resto del país. Aun cuando el uso de fertilizante sin herbicida produce un volumen levemente menor y por ser de menor costo tiene potencial para ser el más económico, el análisis marginal se concentra en la alternativa herbicida-fertilizante (Cuadro $\mathrm{N}^{\circ} 8$ ) a objeto de comparar sus resultados con el resto del país.

La observación más global que se desprende de los resultados es el alto costo que representan las plantaciones espaciadas a $2 \times 2 \mathrm{~m}$ con respecto a las de $3 \times 3 \mathrm{~m}$. Es evidente que tratar 2.500 plantas por hectárea con herbicida y fertilizante es mucho más caro que hacerlo con densidades de 1.111 plantas por hectárea. Este mayor costo, más que el volumen, determina prioritariamente el costo y precio de equilibrio.

La rentabilidad de los tratamientos está asegurada, excepto a densidades de $2 \times 2 m$ y con rotaciones de 10 años, dado que el precio corriente de mercado para la madera en pie supera actualmente los $\$ 4.200 / \mathrm{m}^{3}$ (US $\left.\$ / 12 / \mathrm{m}^{3}\right)$. 


\section{Cuadro $\mathrm{N}^{\circ} 8$}

\section{ANALISIS FINANCIERO MARGINAL PARA EL TRATAMIENTO DE HERBICIDAFERTILIZANTE EN SUELO DE VEGA $(3 \times 3 \mathrm{~m})$}

\begin{tabular}{|c|c|c|c|c|c|c|}
\hline \multirow[t]{2}{*}{ Año } & \multirow[t]{2}{*}{ Gastos de Operación } & \multirow{2}{*}{$\begin{array}{l}\text { Costo } \\
\text { Actual } \\
\text { (\$/ha) }\end{array}$} & \multicolumn{2}{|c|}{$\begin{array}{l}\text { Costo } \\
\text { Año } 10\end{array}$} & \multicolumn{2}{|c|}{$\begin{array}{c}\text { Capitalizado } \\
\text { Ano } 15\end{array}$} \\
\hline & & & $8 \%$ & $12 \%$ & $8 \%$ & $12 \%$ \\
\hline 0 & $\begin{array}{l}\text { Dosis de herbicida } \\
0.5 \text { jornadas+leyes } s 0 c \text {. } \\
1.5 \mathrm{~L} \text { Round-up }(\$ 9000 / \mathrm{L}) \\
20 \% \text { dosis fertilizante } \\
35.1 \mathrm{Kg} \text { /ha Urea }(\$ 96 / \mathrm{Kg}) \\
16.7 \mathrm{Kg} / \text { ha Super } \mathrm{f} \text { triple } \\
12.0 \mathrm{Kg} / \text { ha Borax }(\$ 181 / \mathrm{Kg}) \\
1 \text { jornada+leyes } s 0 c \text {. } \\
1 / 5 \text { jornadas+leyes } s 0 c \text {. }\end{array}$ & $\begin{array}{r}2.000 \\
13.500 \\
3.370 \\
1.416 \\
2.172 \\
4.000 \\
1.800\end{array}$ & & & & \\
\hline 1 & $\begin{array}{l}40 \% \text { dosis fertilizante } \\
70.2 \mathrm{Kg} \text { ha Urea }(\$ 96 / \mathrm{Kg}) \\
33.4 \mathrm{Kg} / \text { ha Super f triple } \\
24 \mathrm{Kg} \text { /ha Borax } \\
2 \text { jornadas+leyes soc. } \\
1 / 5 \text { jornadas de superv. }\end{array}$ & $\begin{array}{r}28.258 \\
6.740 \\
2.839 \\
4.344 \\
8.000 \\
3.600\end{array}$ & 61.007 & 87.765 & 89.639 & 15.4672 \\
\hline \multirow[t]{2}{*}{2} & $\begin{array}{l}40 \% \text { dosis fertilizante } \\
70.2 \mathrm{Kg} / \mathrm{ha} \text { Urea }(\mathrm{SS} 6 / \mathrm{Kg}) \\
33.4 \mathrm{Kg} \text { ) } \\
24.0 \mathrm{Kg} \text { Super f triple } \\
2 \text { jornadas +leyes soc. } \\
1 / 5 \text { jornada supervision. }\end{array}$ & $\begin{array}{r}25.523 \\
6.740 \\
2.839 \\
4.344 \\
8.000 \\
3.600\end{array}$ & 51.020 & 70.777 & 74.966 & 124.734 \\
\hline & & 25.523 & 47.241 & 63.194 & 69.413 & 111.369 \\
\hline & & Gran Total & 159.268 & 221.736 & 234.018 & 390.775 \\
\hline \multicolumn{3}{|c|}{$\begin{array}{l}\text { Costo de equilibrio } \$ / \mathrm{m}^{3} \text { en pie } \\
\text { Beneficio Neto=tasa de interés }\end{array}$} & $\begin{array}{r}2.250 \\
+180\end{array}$ & $\begin{array}{r}3.132 \\
376\end{array}$ & $\begin{array}{r}1.219 \\
98\end{array}$ & $\begin{array}{r}2.036 \\
244\end{array}$ \\
\hline \multicolumn{3}{|c|}{ Precio de Equilibrio $\$ / \mathrm{m}^{3}$ en pie } & 2.430 & 3.508 & 1.317 & 2.280 \\
\hline
\end{tabular}

\section{CONCLUSIONES Y RECOMENDACIONES}

La preparación del sitio, entendiendo como tal el método de plantación, el 
control de las malezas y/o fertilizaciones de apoyo, es imprescindible para lograr el éxito en el establecimiento. Su importancia se debe al marcado efecto en el crecimiento y la supervivencia cuando las plantas son más vulnerables.

Hay empresas en la zona costera-sur de la provincia de Concepción que han perfeccionado técnicas complejas de preparación del sitio que incluyen, además de las faenas señaladas, el uso de micorrizas y riego (Bourke M., 1991 comn. pers.). Combinando esta preparación con una selección de la época de plantación (Octubre, Noviembre y Diciembre) se logran resultados sobresalientes.

Los ensayos muestran que la ahoyadura con motoperforadora es una técnica inadecuada para suelos con cierto grado de arcillas; ya que produce, por compresión, un efecto de maceta en las paredes del hoyo. El método tradicional, de ahoyadura con pala sigue siendo el mejor, aun cuando no difiere significativamente del tratamiento de casillas o surcos.

La interacción del espaciamiento en el tratamiento de herbicida y/o fertilizante se advierte más en el aumento del costo que en el incremento del volumen. En esta forma, dado que el análisis económico es de tipo marginal, las densidades bajas $(3 \times 3 \mathrm{~m})$ resultan económicamente más deseables que 2 $\times 2 \mathrm{~m}$. Un análisis económico que considere el costo total e ingreso total, puede variar esta última conclusión.

De todas maneras, el tratamiento de fertilización en espaciamientos de $2 \mathrm{x}$ $2 \mathrm{~m}$ en rotaciones de 15 años y el de herbicida-fertilización en $3 \times 3 \mathrm{~m}$ resultan económicamente factibles, pues necesitan de precios de mercado menores de US\$ $12 / \mathrm{m}^{3}$ (margen inferior del rango actual) para ser rentables a un $12 \%$ de interés.

Una forma de reducir los costos del tratamiento herbicidas es hacer la aplicación localizada en círculos alrededor de la planta y no en fajas. Al reducir la superficie tratada al mínimo se consigue igual beneficio con un consumo de herbicida menor.

Esta técnica es segura en zonas áridas donde la densidad y desarrollo del pasto es reducida y se estima de factibilidad limitada en las zonas más lluviosas (Prado, J.A. y Wrann J., 1988).

Es recomendable también estudiar nuevas dosis reducidas de fertilizantes, para establecer a menor costo las dosis minimas que resulten igualmente efectivas. 
Finalmente se recomienda que para ensayos como este, que producen un incremento del volumen, se documenten también los cambios en el tamaño de trozas, calidad del producto y cambio en los costos productivos. Estos efectos laterales tienen una importancia igual o mayor que el aumento de volumen; único factor considerado en el análisis marginal.

\section{BIBLIOGRAFIA}

Goodwin N. y Candy S.G., 1986. Single-tree and Stand Growth Models for a Plantation of Eucalyptus globulus Labill. in Northern Tasmania. Aust. For. Res. 16. 131-44.

INFOR., 1988. IV Informe de Actividades Período: Abril 1987 -Junio 1988. Proyecto Manejo Silvicola del Género Eucalyptus. CORFO.

Prado J.A. y Rojas P., 1987. Preparación del Sitio y Fertilización en el Establecimiento de Plantaciones de Eucalyptus globulus en la Zona Semiárida de Chile. Ciencia e Investigación Forestal Vol. $1 \mathrm{~N}^{\circ} 1: 17-27$.

Prado J.A. y Barros S., 1989. Eucalyptus. Principios de Silvicultura y Manejo INFOR. CORFO Santiago, Chile.

Prado J.A. y Wrann J., 1988. La Importancia de la Preparación del Sitio y la Fertilización en el Establecimiento de Plantaciones de Eucalyptus. IV Informe de Actividades. Período Abril 1987 - Junio 1988. Proyecto Manejo Silvícola del Género Eucalyptus. INFOR Santiago-Chile 1988.

Wrann J. e Infante P., 1988. Métodos para el Establecimiento de Plantaciones de Eucalyptus camaldulensis y Quillaja saponaria en la Zona Arida de Chile. Ciencia e Investigación Forestal. Vol $2 \mathrm{~N}^{\circ} 1$. 


\section{APENDICE $N^{\circ} 1$}

COMPARACION DEL VOLUMEN DEL ARBOL MEDIO A LOS

5 AÑOS DE EDAD

$$
\left(\mathrm{m}^{3} \mathrm{sec}\right)
$$

\begin{tabular}{|l|c|c|c|c|}
\hline Espaciamiento & \multicolumn{3}{|c|}{$\begin{array}{c}\text { Zona Costera-Sur } \\
\text { Prov. Concepcion }\end{array}$} & $\begin{array}{c}\text { Northern Tasmania } \\
\text { Australia }\end{array}$ \\
\hline \multirow{2}{*}{$2 \times 2 \mathrm{~m}$} & Lisboa & Diaz & Promedio & Promedio \\
\cline { 2 - 5 } & & & & \\
Fertilizante & 0,011126 & 0,033440 & 0,022283 & 0,022490 \\
Testigo & 0,005781 & 0,016557 & 0,011169 & 0,011136 \\
\hline $3 \times 3 \mathrm{~m}$ & & & & \\
Herb-Fert. & 0,023011 & 0,045308 & 0,034160 & 0,030473 \\
Testigo & 0,002521 & 0,019812 & 0,011167 & 0,013325 \\
\hline
\end{tabular}

